\title{
Equipos negociadores y cobertura de las agendas climáticas en las COP: el caso de Argentina entre 2012 y 2019
}

\author{
María del Pilar Bueno* \\ Patricio YAMIN VÁZQUeZ** \\ JOÉL HERnÁN GONZÁLEZ***
}

Artículo recibido: 30 de junio de 2021

Artículo aprobado: 22 de noviembre de 2020

Doi: https://doi.org/10.12804/revistas.urosario.edu.co/desafios/a.10760

Para citar este artículo: Bueno, M. P., Yamin Vázquez, P. Y., \& González, J. H. (2022). Equipos negociadores y cobertura de las agendas climáticas en las COP: el caso de Argentina entre 2012 y 2019. Desafíos, 34(1), 1-36. https://doi.org/10.12804/revistas.urosario.edu. co/desafios/a.10760

\section{Resumen}

La complejización de la gobernanza climática internacional en el contexto de la Convención Marco de Naciones Unidas sobre Cambio Climático ba generado que los Estados enfrenten una creciente demanda de recursos humanos especializados para afrontar los espacios de negociación en las Conferencias de las Partes (COP). A partir de la caracterización de las delegaciones argentinas en las COP de 2012 a

\footnotetext{
* Correo electrónico: maria.bueno@ffcpolit.unr.edu.ar. ORCID: https://orcid.org/00000003-3408-6271

** Correo electrónico: pyaminvazquez@udesa.edu.ar. ORCID: https://orcid.org/00000002-9964-3430

*** Correo electrónico: joel.hernan.gr@gmail.com. ORCID: https://orcid.org/0000-00015667-5566
} 
2019, se identificó al grupo negociador y luego se examinaron los temas de las agendas cubiertos por dichos equipos, conforme a un proceso de agrupamiento. Se afirma que existe una relación entre la cobertura de los temas y los intereses del Estado con relación a su politica climática, lo que se refuerza en los paises del Sur Global. El artículo presenta información inédita recabada, principalmente, a través de técnicas cualitativas y se asienta en un proceso de revisión de la literatura sobre delegaciones nacionales en conferencias climáticas.

Palabras clave: cambio climático; negociaciones internacionales; delegaciones nacionales; agendas; Acuerdo de París; Argentina.

\title{
Negotiation Teams and Coverage of Climate Agendas in COPs: The Case of Argentina between 2012 and 2019
}

\begin{abstract}
As a consequence of the incremental complexity of international climate governance, particularly within the United Nations Framework Convention on Climate Change, States face an increasing demand on specialized human resources to participate in different negotiation settings during the Conferences of the Parties (COPS). We characterize the Argentinian delegations between 2012 - 2019, identify the negotiation team and analyze the coverage of the team's agenda. Interests regarding climate change international politics can be inferred from the participation or absence of negotiators within different discussions. This paper reviews previous empirical work on delegations in climate change negotiations and draws upon qualitative techniques in order to collect original data to perform an in-depth case analysis.
\end{abstract}

Keywords: Climate change; international negotiations; national delegations; agendas; Paris Agreement; Argentina. 


\title{
Equipes de negociação e cobertura das agendas climáticas na COP: o caso da Argentina entre 2012 e 2019
}

\begin{abstract}
Resumo
A complexidade da governança climática internacional no contexto da Convenção-Quadro das Nações Unidas sobre Mudanças do Clima tem feito com que os Estados enfrentem uma demanda crescente por recursos humanos especializados para faz̧er frente aos espaços de negociação na Conferências das Partes (COP). A partir da caracterização das delegações argentinas na COP no periodo de 2012 a 2019, o grupo de negociação é identificado e, a seguir, os temas das agendas das referidas equipes são examinados por meio de um processo de agrupamento. Afirma-se que existe uma relação entre a abrangência das questões e os interesses do Estado em relação à sua política climática, a qual é reforçada nos países do Sul Global. O artigo apresenta informações inéditas coletadas, principalmente, por meio de técnicas qualitativas, e se baseia em um processo de revisão da literatura sobre delegacõos nacionais em conferências sobre o clima.
\end{abstract}

Palavras-chave: mudanças climáticas; negociações internacionais; delegacoôes nacionais; agendas; Acordo de Paris; Argentina.

\section{Introducción}

La gobernanza climática internacional ha estado sujeta a importantes transformaciones en las últimas décadas como producto de la aceleración de la urgencia y de la brecha creciente de ambición de mitigación, adaptación y financiamiento informada por la ciencia y otros organismos internacionales (IPCC, 2018; PNUMA, 2020, PNUD, 2021). Asimismo, tales cambios se vinculan con dos desafíos adicionales e imbricados: la complejidad del proceso negociador en el régimen climático de la Convención Marco de Naciones Unidas sobre Cambio Climático (CMNUCC) en términos temáticos y la incorporación de un conjunto de actores no estatales y subnacionales (Andonova et al., 2009; Bulkeley et al., 2012). Cabe resaltar que la complejidad del debate climático, incluyendo su carácter técnico y la ampliación de su alcance, no es un fenómeno reciente, sino que ha estado presente 
desde las instancias iniciales de las negociaciones, como muestra el análisis de los primeros años de estas (Grub \& Yamin, 2001). No obstante, estos procesos se han profundizado, generando una situación en la que los Estados enfrentan una creciente demanda de recursos humanos cada vez más especializados para poder afrontar todos los espacios de negociación existentes. Asimismo, la multiplicidad de temas y actores estatales y no estatales contenidos dentro de los mecanismos de gobernanza climática plantean un desafío creciente en términos de los modos tradicionales de ejercer la diplomacia y la negociación. Estos desafíos son particularmente relevantes para los Estados del Sur Global, quienes en términos relativos disponen de menores recursos y capacidades para hacer frente a las negociaciones, lo que genera una situación de inequidad donde a menudo se debe privilegiar la participación en algunos espacios dentro de las Conferencias de las Partes (COP) a unos conjuntos de actores y dejar de lado otros (Martinez et al., 2019). En este contexto, el estudio de la composición y accionar de las delegaciones de los países en desarrollo cobra una particular relevancia para identificar el accionar y las preferencias en las negociaciones climáticas.

A partir de esto, el presente trabajo busca responder a la siguiente pregunta: ¿cómo se vincula la composición de la Delegación Argentina (DA) con la cobertura que el país logró con relación a los temas tratados en las COP? El periodo que se analiza es el comprendido desde la COP de Doha en 2012 hasta la última celebrada en Madrid en 2019. Este puede ser analizado como una unidad en tanto se extiende durante todo el proceso de negociación del Acuerdo de París, así como de su implementación. El hecho de que existan escasos antecedentes sobre la composición de la DA en las COP aporta originalidad a la propuesta de este artículo (Bueno et al., 2021). A la vez, aquí se avanza en un análisis sobre un subconjunto específico dentro de la delegación: el equipo negociador. De este modo, el propósito que se plantea este trabajo es doble: además de profundizar en el conocimiento sustantivo sobre Argentina y su participación en las negociaciones climáticas, se busca mostrar la relevancia y el potencial aporte que pueden hacer estudios cualitativos en profundidad sobre las delegaciones y su participación para la comprensión del accionar de los Estados dentro de 
la negociación, especialmente en relación con aquellos elementos a los que asignan mayor relevancia dentro de la misma.

Cabe notar que, de acuerdo con las reglas de procedimiento de la CMNUCC (FCCC/CP/1996/2), los Estados parte pueden nombrar una delegación compuesta por un jefe o jefa de delegación, representantes con acreditación, representantes alternos y asesores. En el registro de delegados/as que completa el punto focal de cada Estado se incluyen las siguientes categorías: jefe/a de delegación; jefe/a de delegación alterno/a; representantes (Party) y asesores/as (Party overflow). Las credenciales asignadas a las tres primeras categorías habilitan a la representación en los distintos espacios que componen la negociación; quienes cuenten con una credencial de asesor o Party overflow no podrán hacer uso de la palabra en nombre del país, excepto con permiso expreso del/de la jefe/a de delegación. De este modo, estudiar a los primeros tres grupos toma una importancia central para poder profundizar sobre los intereses de los Estados dentro de la negociación.

En este punto, el trabajo presenta información inédita recabada, principalmente, a través de técnicas cualitativas. El análisis general de la DA fue realizado a partir de las listas oficiales de participantes, disponibles en el sitio web de la CMNUCC. ${ }^{1}$ Aquí se recolectó la información referida a la DA, incluyendo su rol y área de gobierno a la que pertenecía cada persona allí incluida. Por su parte, el análisis específico sobre el equipo negociador y su participación en las negociaciones se confeccionó a partir de entrevistas y observación participante durante todas las COP del periodo estudiado. Para el presente trabajo fueron entrevistados como informantes clave cinco representantes que han tenido una reiterada participación en el equipo negociador y que, por lo tanto, pudieron brindar información de relevancia para identificar no solo qué subconjunto de personas cumplieron las funciones de

\footnotetext{
1 Se reconoce que las listas tienen limitaciones, en la medida que no figuran los participantes con el estatus de Party overflow y, en ocasiones, hay errores de actualización en el estatus de los participantes. Sin embargo, representan el único registro formal de conformación de las delegaciones.
} 
equipo negociador, sino también a qué espacios específicos dentro de la negociación fueron asignados. Esto último representaba un desafío, en tanto que la agenda de la negociación, si bien mantiene algunos puntos estables en el tiempo, difiere en cada conferencia. Con el objeto de poder hacer una comparación a lo largo del tiempo, se procedió al agrupamiento a partir de áreas temáticas para cada punto de las agendas de las COP y los órganos subsidiarios durante todo el periodo estudiado.

Para lograr estos objetivos, el trabajo se dividió en tres partes. En primer lugar, se revisó la literatura previa sobre la relación entre las delegaciones, su conformación y la capacidad diplomática de los Estados en las negociaciones climáticas. En este punto se identificó la existencia de una fuerte limitación, en tanto se observa que los trabajos empíricos realizados previamente han utilizado enfoques cuantitativos, dejando de lado las aproximaciones cualitativas sobre los casos, tal como la que se propone en el presente estudio.

En segundo lugar, se analizaron las características y la composición de la DA. En este caso, la abordamos a partir de dos niveles de análisis: por un lado, en línea con la literatura previa, la delegación en su totalidad; por el otro, el equipo negociador como el subconjunto específico que tiene potestades en nombre del Estado que representa. Este análisis abarcó la evolución en su composición y la representación de diferentes instituciones, incluyendo cada una de las áreas de gobierno involucradas, así como otros actores, entre ellos: representantes provinciales o legisladores.

En tercer lugar, el trabajo identificó la cobertura de la agenda climática alcanzada por el país en el periodo de estudio. Para esto, se tomaron en cuenta las agendas de cada una de las COP y de los órganos subsidiarios permanentes y ad hoc. Dichos temas se aglutinaron de modo que permitieran reconstruir la representación en cada uno, a partir de los testimonios de diferentes participantes dentro de la negociación. El resultado de este trabajo nos permitió observar la participación de Argentina en 21 áreas: mitigación; adaptación; pérdidas y daños; financiamiento; tecnología; construcción de capacidades; transparencia; balance mundial; cumplimiento e implementación; 
medidas de respuesta; países menos avanzados; agricultura; mercados; ACE (educación, sensibilización y comunicación en sus inicios); bosques; aspectos ligados a la ciencia; aspectos metodológicos bajo CMNUCC; género; otros temas de implementación del AP; Plataforma de Comunidades Locales y Pueblos Indígenas (LCIPP, por sus siglas en inglés), y marcos temporales comunes o common time frames. En este punto, se buscó vincular algunas características del equipo negociador con la capacidad del país de mantener representación en los diferentes espacios que conforman la negociación, junto con rasgos de la política climática nacional y el propio contexto de la CMNUCC.

\section{Delegaciones y negociaciones climáticas}

La mayor parte de los trabajos realizados presentan un enfoque cuantitativo basado en las listas oficiales de participantes, que constituyen una fuente primaria muy valiosa para poder reconstruir el objeto de estudio. Los trabajos se han enfocado en dos aspectos diferenciados: las consecuencias que los diferentes tipos de delegaciones tienen sobre la capacidad del Estado en las negociaciones, especialmente en el caso de actores con pocos recursos, y los factores que permiten explicar la notable variación y las diferentes dimensiones de las delegaciones nacionales.

Al estudiar el impacto de presentar delegaciones numerosas y con recursos humanos calificados, la literatura asume que esto otorgaría una mayor capacidad de negociación a los Estados. Existen importantes razones para considerar esto: delegaciones más grandes y mejor preparadas permiten que el país tenga una mayor representación en más espacios dentro de la negociación, así como mayor capacidad para construir redes formales e informales para influenciar resultados (Kaya \& Schofield, 2020; Lövbrand et al., 2017; Schroeder et al., 2012).

Cabe resaltar que si bien la dimensión y el nivel de preparación de las delegaciones es solo uno de los muchos factores que pueden influir en el éxito de un país en una negociación (Bailer, 2017), la literatura señala que estos aspectos son cruciales para los Estados más pequeños 
del Sur Global que, dada la escasez de recursos, a menudo enfrentan problemas para cubrir la creciente cantidad de temas en agenda y eventos paralelos, para hacer frente a las notablemente extensas jornadas de negociación o superar las barreras lingüísticas, especialmente en el caso de países no angloparlantes (Kaya \& Schofield, 2020; Martinez et al., 2019; Schroeder et al., 2012; Stevenson \& Dryzek, 2014). Esto ha llevado al desarrollo de trabajos abocados a estudiar las estrategias mediante las cuales los Estados en desarrollo buscan superar esta situación de inequidad, incluyendo la negociación a partir de coaliciones regionales o temáticas, que permiten ganar peso en la negociación, o alianzas con Organizaciones de la Sociedad Civil (OSC), que a menudo aportan información o conocimientos técnicos a las delegaciones (Betzold, 2010; Bhandary, 2017; de Águeda Corneloup \& Mol, 2014; Ourbak \& Magnan, 2018; Roger \& Belliethathan, 2016). Con relación a este último punto, se observó una tendencia creciente a la inclusión de OSC como parte de las delegaciones nacionales, especialmente en el caso de Estados de bajo nivel de ingresos, como los países africanos en la etapa posterior a la COP15 de Copenhague, aunque no solamente restringida a este grupo (Roger \& Belliethathan, 2016; Schroeder et al., 2012; Skovgaard et al., 2019). La presencia de técnicos de OSC altamente capacitados en las delegaciones nacionales puede dar lugar incluso a que ocupen un rol preponderante dentro de las mismas (Fisher \& Green, 2004). Cabe resaltar, además, que la desigualdad entre las delegaciones ha sido también objeto de atención de la Secretaría Ejecutiva de la CMNUCC, que ha buscado mitigar sus efectos a partir de diferentes acciones, como la interpretación simultánea en todas las reuniones formales, la limitación a la cantidad de eventos en paralelo y el financiamiento de dos delegados para todos los países pertenecientes al grupo de los países menos desarrollados, aunque los efectos de estas iniciativas han sido limitados (Stenveson \& Dryzek, 2014, p.76).

En consecuencia, las negociaciones climáticas se encuentran marcadas por la inequidad que se deriva de los desiguales recursos financieros, técnicos y humanos. Esto afecta directamente la capacidad negociadora de los Estados, entendida como la posibilidad de influir y obtener resultados favorables a sus intereses (Martinez et al., 2019; Weiler, 
2012). ${ }^{2}$ De este modo, no es sorprendente que la disponibilidad de recursos de un país haya sido un factor clave a la hora de explicar la composición de las delegaciones: los Estados que cuentan con mayor cantidad de recursos materiales y humanos han logrado conformar delegaciones más numerosas. Cabe resaltar, no obstante, que algunos trabajos empíricos sugieren que no existe una relación lineal entre ambos factores, sino que más allá de un determinado umbral, un aumento de los recursos financieros no necesariamente incrementa la delegación (Kaya \& Schofield, 2020). Este punto refuerza el argumento presentado anteriormente: la disponibilidad de recursos impacta fuertemente sobre los países pequeños y medianos, pero no necesariamente sobre los más poderosos.

El nivel de desarrollo también se encuentra correlacionado con el tipo de integrantes dentro de las delegaciones. En este punto, estudios previos muestran que la representación de los países desarrollados suele estar dominada por las carteras de ambiente, mientras que las de los países en desarrollo cuentan con mayor presencia en las áreas económicas o de relaciones exteriores (Skovgaard et al., 2019). Esto no es sorprendente, en tanto los reclamos históricos del Sur Global han estado asociados a la concepción del cambio climático desde una perspectiva de desarrollo y a la ampliación de la agenda desde la mitigación hacia otros temas como la adaptación, el financiamiento y el apoyo para una transición a una economía baja en carbono (Joshi, 2015).

Un segundo factor relevante para entender la participación de los Estados en las negociaciones es el interés presentado sobre las mismas. Este punto puede ser analizado tanto en términos de variación temporal como entre los casos. Por un lado, momentos clave dentro de las negociaciones suelen estar correlacionados con una mayor participación de los Estados tanto en términos del número de integrantes

2 Cabe señalar que Weiler (2012) no encuentra efectos significativos de la medida de la delegación sobre el éxito en la negociación, pero sí los encuentra para el poder de los Estados, medido como el logaritmo natural del producto interno bruto (PIB). En este sentido el modelo de Weiler captura la desigualdad de recursos que se menciona en el presente trabajo y corrobora el efecto sobre la capacidad negociadora de los Estados. 
de las delegaciones, como en la cantidad de carteras de gobierno en ellas representados (Skovgaard et al., 2019). Esto parecería indicar delegaciones que buscan poder cubrir una mayor cantidad de temas, tal como se deriva del número de personas y de los intereses que estas representan.

Por otro lado, aquellos países que asignan una importancia alta a la negociación también tienden a presentar delegaciones más grandes. Así, países con grupos de interés domésticos que se vean afectados por las consecuencias del cambio climático o de las políticas de descarbonización tienden a presentar delegaciones más numerosas. Así lo observan Kaya y Schofield (2020): el caso de países con presencia de industrias amenazadas por las políticas contra el cambio climático como el sector de combustibles fósiles. Si bien no se encuentran resultados concluyentes, la vulnerabilidad también podría afectar el número y composición de las delegaciones, especialmente a partir de eventos climáticos extremos que modifican las percepciones de los tomadores de decisiones y fortalecen la demanda de actores no estatales y de los propios negociadores (Roger \& Belliethathan, 2016).

El tipo de régimen político, por su parte, es también un factor explorado. Diversos trabajos han identificado un efecto positivo de la democracia tanto sobre el número de integrantes de las delegaciones (Kaya \& Schofield, 2020), como sobre la incorporación de la sociedad civil (Böhmelt, 2013) o participación más equitativa en términos de género (Kruse, 2014).

Por último, cuestiones logísticas también parecen afectar el resultado. En este punto, el hecho de ser anfitrión de una COP, el atractivo de la sede o la distancia geográfica entre la misma y el Estado que envía la delegación también afectan el número de representantes que este último puede enviar (Kaya \& Schofield, 2020; Schroeder et al., 2012).

No obstante su creciente desarrollo, el estudio acerca del rol de las delegaciones presenta algunas limitaciones. En primer lugar, los trabajos se han basado principalmente en enfoques cuantitativos que utilizaron como insumo los listados oficiales de participantes provistos 
por la Secretaría de la CMNUCC que no diferencian aquellos integrantes de las delegaciones que son negociadores de los que no (Kaya \& Schofield, 2020; Kruse, 2014; Martinez et al., 2019; Schroeder et al., 2012). Asimismo, la definición acerca de quiénes deberían ser incluidos oficialmente en las delegaciones ha estado marcada tanto por cambios temporales en el registro oficial, como nacionales en términos de los criterios utilizados. Esto hace extremadamente dificultoso poder diferenciar claramente a gran escala con cuántos negociadores ha contado cada Estado parte en cada conferencia. En contrapartida, los estudios cualitativos sobre esta cuestión, si bien podrían profundizar más sobre esta distinción y sobre las características personales de esos negociadores, han sido notablemente escasos.

En segundo lugar, los trabajos que han abordado en detalle casos específicos han tendido a obviar a los actores de peso medio en la negociación. En este sentido, la mayor parte de esta literatura ha privilegiado el estudio de los Estados más pequeños y las limitaciones que estos enfrentan (Martinez et al., 2019; Schroeder et al., 2012). En el otro extremo, existe también producción académica, aunque en menor número, que ha puesto el foco sobre los actores de mayor peso dentro la negociación (Biedenkopf \& Petri, 2019; Skovgaard et al., 2019).

El presente trabajo toma como punto de partida estas limitaciones. No solo se presenta aquí un caso no abordado previamente en la literatura, sino que además se profundiza sobre la composición de la DA, incluyendo tanto el análisis general como específico del equipo negociador, lo que permite ir un paso más allá e identificar cómo ha sido la cobertura del país en relación con los puntos de la agenda. Esto es central en el argumento del presente trabajo: sin el estudio en profundidad del equipo negociador y su accionar en las negociaciones no es posible deducir del análisis general de la DA las áreas de mayor interés para el Estado. En consecuencia, se entiende que existe una relación entre la cobertura de los temas de la agenda climática y el interés del Estado con relación a su política climática, lo que se refuerza en el caso de los países del Sur Global, teniendo en cuenta los escasos recursos para financiar delegaciones más numerosas. 


\section{Delegación Argentina: evolución y particularidades}

La evolución en la relevancia del cambio climático como tema de la agenda internacional y de la CMNUCC como espacio articulador y orquestador de tal gobernanza (Abbott, 2017) puede visualizarse tanto en términos cualitativos (acuerdos alcanzados por las Partes, ampliación y complejización de la agenda climática) como cuantitativos. Esto último se observa, entre otras cuestiones, en el incremento del número de Estados parte de la CMNUCC, la mayor cantidad de delegados de las Partes en las COP y su mayor participación en el número total de acreditados. Entre la primera COP desarrollada en Berlín y la COP25 de Madrid, el número de Estados parte se incrementó de 117 a 196, los delegados de los países pasaron de 757 a 11406 y su participación en el número total de asistentes creció del 19 al 51\% (Bueno et al., 2021). Si bien la evolución histórica del tamaño de las delegaciones es clara, esto no implica linealidad. Al respecto se registraron picos de participantes en la COP15 de Copenhague y la COP21 de París, en tanto que otras como la COP19 mostraron una reducción considerable en las delegaciones. Esto obedece a distintos factores vinculados a las posibilidades materiales de los Estados, la ubicación geográfica del anfitrión y, sobre todo, las expectativas políticas en torno al alcance y ambición de los compromisos. Esto último es particularmente evidente en los casos de París y Copenhague; esta última, a pesar de su fracaso, llegó con altas expectativas y optimismo en torno al alcance de acuerdos.

En este contexto, resulta interesante abordar el comportamiento de la DA. Cabe mencionar que la DA está compuesta por todas las personas inscritas en el registro nacional por el punto focal, que figuran en las listas oficiales de la UNFCCC, sin importar su rol. A pesar de que una gran delegación no necesariamente implica un mayor número de negociadores/as, lo cierto es que puede esgrimirse como indicador valioso. Asimismo, conocer los espacios institucionales de los que provienen los/las delegados/as puede ofrecer información sobre los puntos de la agenda que revisten mayor interés para los Estados y facilita una aproximación hacia la distribución de las cargas relativas 
al proceso negociador. Para ello observamos los aspectos relacionados con su evolución numérica y composición tanto en el periodo 1995-2019, como en el recorte 2012-2019.

Respecto a la evolución histórica del tamaño de la DA en el primer periodo, observamos 3 características (figura 1): a) la delegación creció a lo largo de los años a un ritmo inferior al promedio de otras delegaciones nacionales, b) su crecimiento fue oscilante y c) tal crecimiento, en ocasiones, no coincidió con el comportamiento promedio del resto de las delegaciones en las grandes conferencias. Como primera característica decimos que su crecimiento se dio a una velocidad inferior al promedio global: las 6 delegaciones más pequeñas fueron enviadas entre la COP1 y la COP10. Esto encuentra consonancia con el crecimiento de la agenda y la complejización en la estructura de la gobernanza climática internacional. No obstante, su velocidad fue inferior al promedio y desde 2004, la DA ha superado a la media de las delegaciones solo en 2 oportunidades (2010 y 2011). Respecto a las oscilaciones, se puede decir que mientras que en ocasiones el país envió un número de delegados que no superó el dígito, en otras oportunidades su tamaño superó las 3 cifras. Las 2 mayores delegaciones del país respondieron a momentos donde Argentina ofició de anfitrión de la conferencia (COP4 y COP10). Finalmente, la escasa coincidencia entre las grandes delegaciones del país y el promedio global de las delegaciones también es un hecho a resaltar. Al respecto, puede citarse el ejemplo de la COP15 de Copenhague, donde dada su relevancia se produjo un considerable incremento en el tamaño promedio de las delegaciones $(160 \%)$ respecto a la conferencia previa, situación que no se replicó en el caso argentino. 
Figura 1. Evolución histórica del tamaño de la DA frente a la evolución promedio global

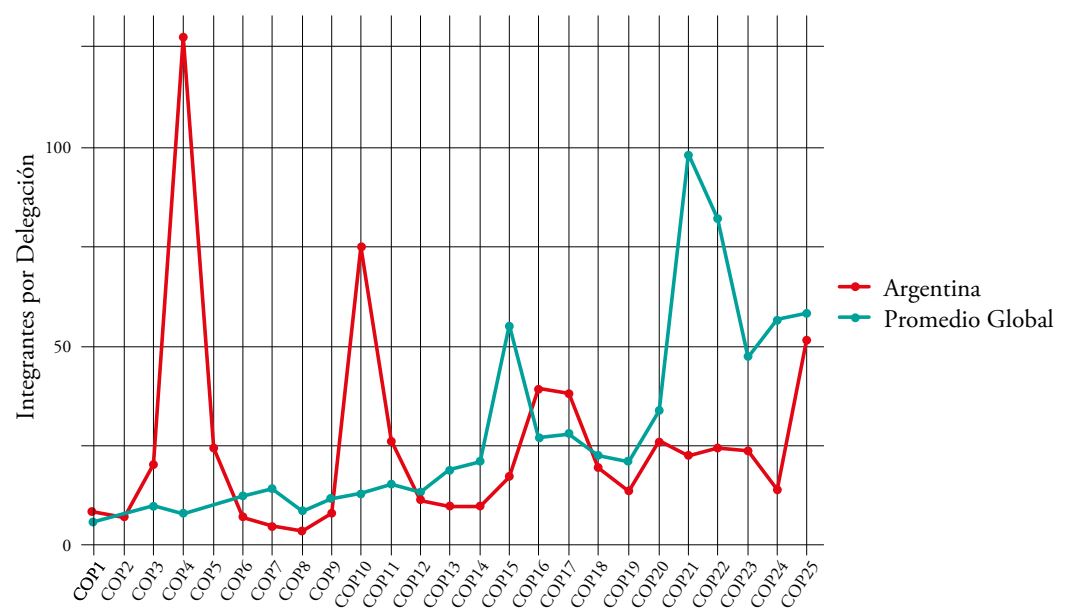

Fuente: elaboración propia.

La oscilación y el desacople del tamaño de la DA encuentran consonancia con vaivenes observados en la política climática Argentina, los cuales no solo han sido resaltados desde un análisis integral de la misma (Bueno, 2012), sino en el análisis de tópicos y programas concretos, como en el caso de los bosques y el programa REDD+ (González, 2018). La política exterior argentina con relación al cambio climático ha estado marcada por un accionar de alto perfil en toda la primera etapa del régimen, lo cual puede observarse en tres factores: la organización de dos COP en Buenos Aires, la evolución de la DA en línea con los cambios globales y el lugar central que ocuparon dentro de la arquitectura climática global algunos de sus integrantes, principalmente el embajador Estrada Oyuela, quien presidió las negociaciones que resultaron en el Protocolo de Kyoto en 1997. En años posteriores, sin embargo, la política exterior climática adoptó posiciones más pasivas y eventualmente defensivas, lo que se evidencia tanto en la mayor distancia entre la evolución de la DA con respecto al promedio global, así como en otros factores que no se incluyen en el presente análisis como su participación en los grupos de negociación (Bueno \& Yamin Vázquez, 2017). 
Respecto a la composición histórica de la DA, el primer elemento que se observa, es que las carteras de ambiente y de relaciones exteriores han sido las predominantes: representan conjuntamente el 79\% de los delegados ministeriales. Esta situación coincidió con su rol como puntos focales ante la CMNUCC. Independientemente de la preponderancia de ambos espacios, sus comportamientos históricos han sido distintos. El área de ambiente, que ha tenido el rango de secretaría y de ministerio en diferentes momentos históricos, muestra una evolución más irregular, no contó con delegados en algunas conferencias, tales como la COP7 y la COP8. Adicionalmente, el comportamiento de ambos espacios permite vislumbrar una tendencia particular, acentuada en el caso de la cartera de relaciones exteriores. Cuanto más grande es el tamaño de la delegación, más diluida se observa la participación porcentual de ambos ministerios en tanto que en casos donde el país presenta delegaciones pequeñas, más protagonismo adoptan ambas carteras. Las COP voluminosas abren el juego a la diversificación de actores en la delegación. En algunos casos, como el de la cop10, esto se tradujo en una mayor presencia de otros poderes del Estado como el legislativo, mientras que en otras, como las COP4 y cOP16, supuso una mayor presencia del resto de las carteras. El rol histórico desempeñado por otras carteras ha sido dispar. No obstante, el área de agricultura ha contado históricamente con un número estable de delegados.

Tabla 1. Conferencias de las Partes en el periodo de estudio (año y sede)

\begin{tabular}{|c|c|c|c|c|c|}
\hline $\begin{array}{c}\text { Número de } \\
\text { COP }\end{array}$ & Año & Ciudad & $\begin{array}{c}\text { Número de } \\
\text { COP }\end{array}$ & Año & Ciudad \\
\hline COP18 & 2012 & Doha & COP22 & 2016 & Marrakech \\
\hline COP19 & 2013 & Varsovia & COP23 & 2017 & Bonn \\
\hline COP20 & 2014 & Lima & COP24 & 2018 & Katowice \\
\hline COP21 & 2015 & París & COP25 & 2019 & Madrid \\
\hline
\end{tabular}

Fuente: elaboración propia.

Por su parte, si observamos las COP desarrolladas durante el recorte temporal 2012-2019 (tabla 1) encontramos que algunas de las mencionadas tendencias se acentúan, en tanto que otras se modifican. Respecto al desacople entre la DA y el promedio global de las delegaciones debemos decir que el mismo se intensifica desde 2012, 
fundamentalmente en las COP21 y COP22. Al igual que ocurrió en Copenhague, en París se produjo un aumento considerable en el tamaño promedio de las delegaciones. No obstante, la DA no acompañó este crecimiento y, de hecho, en París su tamaño se redujo con relación al año anterior. Tal situación convirtió a la COP21 en el mayor momento de desacople entre el tamaño de la DA (23) y la media de las delegaciones (98), con una diferencia de 75 delegados. En este último caso, se puede mencionar que 2015 fue un momento de inflexión nacional por tratarse de un proceso de cambio de signo político en las elecciones nacionales. Sin embargo, esto volvió a suceder en 2019 con distinto comportamiento en el número de asistentes de la DA, puesto que la COP de Madrid fue la tercera más grande. Esto podría deberse a la creciente estabilización temática en la agenda política nacional, incluyendo la sanción de la Ley de Presupuestos Mínimos de Adaptación y Mitigación al Cambio Climático Global a finales de 2019. Asimismo, por primera vez en muchos años, un presidente argentino participó de la COP, lo que involucró un notable incremento de la DA como producto de la comitiva presidencial. Esto evidencia que gran parte de la variación en el número de participantes de la DA en los casos en los que asisten presidentes y vicepresidentes se explica por sus propias comitivas. A su vez, a diferencia de la presidenta Fernández en 2015, Macri en 2019 interpretó a la COP como una caja de resonancia internacional para dar cuenta de sus políticas en un contexto de salida.

Respecto a la pertenencia institucional, se observa el sostenimiento de las carteras de relaciones exteriores y de ambiente como los principales espacios dentro de la DA (figura 2). El área de relaciones exteriores muestra un comportamiento mayormente regular entre las COP. La cartera de ambiente, por su parte, tiene mayores variaciones que apuntan a un aumento de delegados/as de esta cartera, consolidando un crecimiento durante el periodo. Luego de estas dos áreas, la mayor participación ha sido de agricultura, que muestra una presencia ininterrumpida de representantes durante todo el periodo. Por su parte, las áreas de energía, industria, transporte y economía han también tenido representantes en diferentes momentos del periodo. $\mathrm{Al}$ respecto, podría inferirse que la participación en estos espacios y 
el predominio de la agricultura encuentra consonancia con la matriz de emisiones de Argentina y el dinamismo y relevancia de estos sectores en la economía del país.

Cabe destacar que la presencia mayoritaria y constante de los sectores de relaciones exteriores y ambiente se encuentra en línea con la situación en las diferentes delegaciones del mundo (Skovgaard et al., 2019). No obstante, a diferencia de otros países en desarrollo, la participación de la cartera de economía en el caso argentino no ha sido predominante, la COP19 fue la última en contar con delegados y negociadores (tabla 2) en su representación. Asimismo, también es posible observar que la suma de los delegados de los dos ministerios predominantes no ha mostrado grandes diferencias, al menos no en el nivel en que variaba el número total de integrantes de la delegación. En este sentido, en las ocasiones que la DA aumentó su número total esto no ha significado un aumento proporcional de los representantes de las dos principales carteras. Por el contrario, este crecimiento se explica mejor por el aumento de la presencia de otras áreas de gobierno como agricultura y, muy especialmente, por la presencia de grandes números de delegados relacionados con el viaje del vicepresidente (COP20) o del presidente (COP25). Estas comitivas, que incluyen áreas como prensa, equipo médico o seguridad, explican en gran parte las notables variaciones observadas en el periodo, lo que sugiere también cautela sobre el estudio general del número de delegados, en tanto no necesariamente representan una mayor capacidad negociadora del país, ya que se encuentra atado a cuestiones coyunturales y en ocasiones ajenas a las negociaciones.

Tabla 2. Representación por sector o área de la DA (2012-2019)

\begin{tabular}{|l|c|c|c|c|c|c|c|c|}
\hline $\begin{array}{c}\text { Ministerio } \\
\text { o sector } \\
\text { representado }\end{array}$ & COP18 & COP19 & COP20 & COP21 & COP22 & COP23 & COP24 & COP25 \\
\hline $\begin{array}{l}\text { Relaciones } \\
\text { Exteriores }\end{array}$ & 4 & 8 & 11 & 12 & 10 & 10 & 3 & 11 \\
\hline Ambiente & 1 & 2 & 3 & 4 & 4 & 7 & 8 & 8 \\
\hline Agricultura & 2 & 1 & 1 & 1 & 2 & 2 & 2 & 6 \\
\hline $\begin{array}{l}\text { Ciencia y } \\
\text { Tecnología }\end{array}$ & 0 & 0 & 1 & 0 & 0 & 0 & 0 & 0 \\
\hline
\end{tabular}




\begin{tabular}{|l|c|c|c|c|c|c|c|c|}
\hline $\begin{array}{c}\text { Ministerio } \\
\text { o sector } \\
\text { representado }\end{array}$ & COP18 & COP19 & COP20 & COP21 & COP22 & COP23 & COP24 & COP25 \\
\hline Energía & 0 & 1 & 0 & 1 & 2 & 3 & 0 & 0 \\
\hline Economía & 1 & 1 & 0 & 0 & 0 & 0 & 0 & 0 \\
\hline Transporte & 0 & 0 & 0 & 0 & 1 & 1 & 0 & 0 \\
\hline Industria & 0 & 0 & 0 & 0 & 2 & 0 & 0 & 0 \\
\hline Poder Legislativo & 0 & 0 & 0 & 1 & 0 & 0 & 0 & 0 \\
\hline $\begin{array}{l}\text { Representantes } \\
\text { Provinciales }\end{array}$ & 9 & 0 & 0 & 0 & 0 & 0 & 0 & 0 \\
\hline Presidencia & 0 & 0 & 10 & 3 & 2 & 0 & 0 & 24 \\
\hline $\begin{array}{l}\text { Representante } \\
\text { con Cargo }\end{array}$ & 1 & 1 & 0 & 1 & 1 & 1 & 1 & 1 \\
Internacional & 2 & 0 & 0 & 0 & 0 & 0 & 0 & 0 \\
\hline Sindicatos & 0 & 0 & 0 & 0 & 0 & 0 & 0 & 1 \\
\hline ONG
\end{tabular}

Fuente: elaboración propia.

Es también posible observar en la composición de la DA la participación de representantes de otras instituciones por fuera de las carteras nacionales que típicamente integran este espacio. Aquí es posible afirmar, para el caso de la COp18, la inclusión de representantes de la Confederación General del Trabajo, así como una presencia relevante de diferentes organismos provinciales de la Ciudad Autónoma de Buenos Aires, la provincia de Buenos Aires y San Luis. Por su parte, en el caso de los integrantes del Poder Legislativo Nacional, durante el periodo estudiado se observa solamente la participación de un representante en la COp21, el diputado Juan Carlos Villalonga enviado como delegado del gobierno de Mauricio Macri, quien asumió la presidencia durante la celebración de dicha reunión. Cabe aquí destacar que en ocasiones anteriores, la presencia de diputados y senadores en las delegaciones fue más nutrida, especialmente en la COP10 celebrada en el país, pero también en otras como la COP16 que contó con la presencia de 4 legisladores. En el caso de la COp25, también se observó la presencia de la sociedad civil dentro de la delegación.

\section{Grupo negociador: Evolución y particularidades}

En la presente sección nos proponemos analizar la dimensión vinculada al equipo negociador. Como se mencionó en el apartado intro- 
ductorio, es necesario trazar una distinción entre la DA, conformada por todos aquellos acreditados para asistir a las COP y el subgrupo conformado por el equipo negociador.

En el contexto del análisis de la DA, hacemos especial hincapié en el equipo negociador observando también su evolución y composición (figura 2). Como primer aspecto a resaltar podemos mencionar que entre las COP18 y COP25 del total de delegados del país, aproximadamente un $40 \%$ (79/196) conformó el equipo negociador. El número de negociadores/as en las COP analizadas oscila entre 7 y 14 . Al respecto, resulta llamativo el hecho de que no coincidan las dos DA más numerosas en el periodo bajo estudio con aquellas que reunieron un mayor número de negociadores/as; la COP20 de Lima y la COP25 de Madrid, contaron con el número de negociadores más bajo del periodo. Esta discrepancia permite inferir que el tamaño del equipo negociador no se encuentra necesariamente vinculado al tamaño de la DA. Asimismo, la COP21 de París contó con una DA pequeña en relación con otras COP; no obstante, su número de negociadores fue el más abultado del periodo, hecho que encuentra consonancia con la relevancia política de dicha conferencia.

Figura 2. Número de negociadores y áreas de gobierno COP18 - COP25

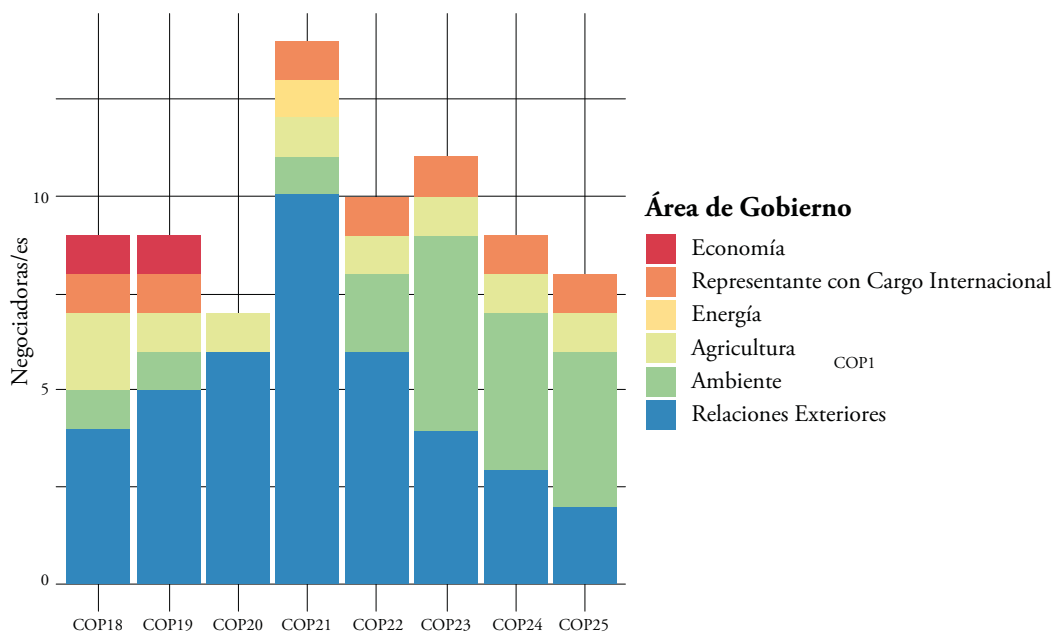

Fuente: elaboración propia. 
Asimismo, el análisis propuesto nos permite observar la reiteración de negociadores a lo largo del tiempo. Los 77 registros como delegados/ as corresponden a 36 negociadores/as, lo que indica una asistencia promedio de 2 cop por cada uno/a de quienes conforman este subgrupo. Sin embargo, la situación es variable y se presentan casos de negociadores/as con un gran número de participaciones y otros/as que lo han hecho en una sola oportunidad. Por un lado, observamos a 9 negociadores/as que han participado en 4 o más COP, mientras que, por el otro, 18 negociadores/as han participado en una única COP en dicho rol. Este elemento es relevante debido a que si bien se registran cambios de negociadores al interior de los tópicos, en ocasiones, como en el caso de tecnología, la cobertura estuvo ligada a la continuidad de un mismo negociador/a a lo largo de las COP.

Respecto a la pertenencia ministerial de los/as negociadores/as, podemos decir que existe consonancia con lo observado en la composición de la DA, hay una primacía de las áreas de relaciones exteriores y de medio ambiente en el proceso. De los 36 negociadores, 17 representaban al área de asuntos exteriores, mientras que 11 la de ambiente. $\mathrm{Al}$ igual que se advirtió en el análisis de composición de la DA, esta última tiene un comportamiento más irregular, aunque se observa en un incremento de sus negociadores desde la COP21 de París, incluso superando en cantidad a los de relaciones exteriores desde la COP23.

Como otro dato relevante, con relación al resto de las carteras, agricultura es la única área de gobierno de las restantes que ha tenido en el periodo analizado un rol relativamente constante en el equipo negociador: apareció en 7 de las 8 COP evaluadas. Este número encuentra consonancia con el análisis general de la DA, donde se lo observa como el tercer espacio con mayor número de delegados. Energía y economía son los otros espacios que han contado con representación, aunque su presencia fue esporádica durante el periodo.

Finalmente, se destaca la participación de representantes con cargos internacionales en la estructura de la CMNUCC que también oficiaron como representantes argentinos en las COP: uno de ellos como presidente del Comité Ejecutivo de Tecnología (TEC) y luego del Centro 
y Red de Tecnología del Clima (СTCN) y otra como copresidenta del Comité de Adaptación. Cabe notar que si bien el primer representante tuvo como pertenencia institucional a una universidad y la segunda tuvo como dependencia a CONICET de 2016 a 2019, en la medida que previamente ofició como negociadora del área de relaciones exteriores, ninguno representó propiamente a instituciones del sistema científico ni tecnológico. Más bien, ambos cuentan con una trayectoria de participación por las carteras ambiental y de asuntos exteriores, respectivamente, sumado al cargo internacional en la estructura de la convención en sus respectivas áreas de especialidad y negociación.

\section{Cobertura argentina de la agenda climática}

En este apartado nos concentramos en dar cuenta de la variable "cobertura de la agenda climática", a sabiendas de que se trata de una construcción conceptual y metodológica. Empezaremos abordando la agenda climática en el contexto de la CMNUCC en el periodo de estudio para luego referir la cobertura de dichos temas por el equipo negociador de la DA.

Agenda climática en la CMNUCC: deconstrucción y aglomeración de temas

$\mathrm{Al}$ aludir a las agendas climáticas en la CMNUCC nos referimos al decálogo de temas, acordados por las Partes de la convención y del Acuerdo de París desde su entrada en vigor (2016), publicado en el sitio oficial que sostiene la Secretaría. Durante el periodo analizado, se tomaron las agendas de las COP de la CMNUCC de 2012 a 2019 y las de los órganos subsidiarios permanentes: el Órgano Subsidiario de Asesoramiento Científico y Tecnológico (SBSTA) y el Órgano Subsidiario de Ejecución o Implementación (SBI). El SBSTA tiene la función de apoyar la labor de la COP y otras conferencias de las Partes como la Conferencia de las Partes del Protocolo de Kyoto (CMP) y la Conferencia de las Partes del Acuerdo de París (CMA), mediante la provisión de información y 
asesoramiento sobre cuestiones científicas y tecnológicas relacionadas con los tres instrumentos. Por su parte, el SBI tiene la función de articular el apoyo a la implementación de los elementos fundamentales de la gobernanza: transparencia, mitigación, adaptación, financiación, tecnología y fomento de la capacidad.

Otras agendas relevadas son las de los órganos subsidiarios conformados con carácter ad hoc. En el periodo de estudio se trata del Grupo de Trabajo Ad Hoc sobre Acción Cooperativa de Largo Plazo (AWG-LCA), establecido mediante la Decisión 1/CP.13 de Bali con el propósito de realizar un proceso comprensivo sobre la implementación de la acción cooperativa de largo plazo, más allá de 2012, que desembocase en un documento para adopción de las Partes en 2009 en Copenhague, que no sucedió. En la práctica, en la cop17 de Durban se negoció un nuevo mandato que alcanzó adopción en París en 2015 y en la COP18 se consideró que el AWG-LCA había finalizado sus deliberaciones.

El AWG-LCA coexistió un año con otro órgano subsidiario ad hoc en el cual se llevaron adelante las tareas técnicas y políticas para lograr el Acuerdo de París, que reflejó el nuevo mandato de negociación: el Grupo de Trabajo Ad Hoc de la Plataforma de Durban (ADP). Con la adopción del Acuerdo de París y las decisiones asociadas se consideró concluida la labor del ADP y se inauguró el Grupo de Trabajo Ad Hoc sobre el Acuerdo de París (APA), cuyo trabajo se extendió de 2016 a $2018 .^{3}$

Cabe mencionar que no se abordan las agendas de la CMP ni los temas relativos al Protocolo de Kyoto en las agendas referidas, en la medida que esta investigación está concentrada en el proceso de París o la acción post- 2020 .

\footnotetext{
3 Por consiguiente, las agendas analizadas para la cobertura son las siguientes. COP18: Agenda COP, SBI, SBSTA, AWG-LCA y ADP. COP19: Agenda COP, SBI, SBSTA y ADP. COP20: Agendas COP, SBI, SBSTA y ADP. COP21: Agendas COP, SBI, SBSTA y ADP. COP22: Agenda COP, CMA, SBI, SBSTA y APA. COP23: Agenda COP, CMA, SBI, SBSTA y APA. COP24: Agenda COP, CMA, SBI, SBSTA y APA. COP25: Agenda COP, CMA, SBI, SBSTA.
} 
Ahora bien, las agendas involucran un conjunto de temas que no necesariamente tienen el mismo peso técnico ni político a la hora de analizar una cobertura temática por delegación. De este modo, hemos realizado un proceso de deconstrucción y aglomeración en base a lo que hemos comprendido como grupos más significativos a partir de las entrevistas realizadas y el proceso de observación participante. Además de la exclusión ligada al PK, no incluimos las consideraciones presupuestarias ni administrativas. Todo el resto de temas formalizados en las agendas fueron incluidos, solo que agrupados bajo un título representativo. Se realizó esta distinción puesto que la CMNUCC es un terreno fértil para la generación de grupos informales sobre diversos temas que no necesariamente figuran en las agendas, porque el hecho de estar en la agenda supone acuerdo de las Partes al efecto. Un ejemplo de mucho peso político para la región de América Latina y el Caribe (ALyC) es el caso de la particular vulnerabilidad de África. Dicha región ha buscado que se le reconocieran en el Acuerdo de París flexibilidades que se le dieron a los países menos desarrollados y a los pequeños Estados insulares en desarrollo de modo infructuoso por el bloqueo oficiado por ALyC. Este punto nunca pudo ser incluido en la agenda por falta de consenso, pero se ha debatido desde antes del recorte temporal y Argentina lo cubrió a un alto nivel de jefe/ a de delegación o de jefe/a alterno/a.

Los grupos informales no solo son un recurso ante la falta de consenso para incluir temas formalmente en la agenda, sino que representan temas emergentes y evoluciones subtemáticas cuando las agendas ya están negociadas. Asimismo, dichos grupos revelan coyunturas políticas y, en tal sentido, se enuncian los casos de los grupos informales conformados en las negociaciones hacia y en París respecto al texto del acuerdo, como el del preámbulo o los aspectos jurídicos. Además, tradicionalmente la Cancillería cubre las negociaciones de cargos o roles en los órganos de la CMNUCC, así como las negociaciones en los grupos regionales como el GRULAC o el G77 más China, entre otros. Ninguno de estos aspectos están incluidos en los grupos, en la medida que solo se aglutinaron los temas formales de las agendas. Otro ejemplo es el de la agenda de bosques que ha figurado formalmente en la agenda del SBSTA como guías metodológicas para 
actividades relativas a la reducción de las emisiones de la deforestación y la degradación forestal y también en otras agendas con otras denominaciones. En la COP21 no figura el tema formalmente en las agendas (figura 3). Sin embargo, esto no quita que no haya habido negociaciones informales que permitieran la inclusión del artículo 5 del Acuerdo de París.

Del mismo modo, existen algunos aspectos que han cambiado de denominación en el periodo y que hemos aglutinado de un modo representativo. Por ejemplo, el tema agricultura se denominó "aspectos ligados a la agricultura" hasta el establecimiento del Trabajo Conjunto de Koronivia en Agricultura en la COP23. A los fines del análisis, lo hemos denominado agricultura.

Por otro lado, la adaptación incluyó los aspectos de pérdidas y daños hasta su división más definitiva en el Acuerdo de París en dos artículos distintos (artículos 7 y 8, respectivamente). Sin embargo, ya en la COP19 se instituyó el Mecanismo Internacional de Varsovia sobre Pérdidas y Daños (WIM) y su Comité Ejecutivo (ExCOM). A los fines de simplificación, hemos considerado ambos temas por separado durante todo el periodo, a pesar de esta distinción política de negociación conjunta hasta 2015.

Si bien al interior de los grupos hubo variaciones, hemos construido tópicos con un nivel de variación relativamente más bajo. A saber: mitigación; adaptación; pérdidas y daños; financiamiento; tecnología; construcción de capacidades; transparencia; balance mundial; cumplimiento e implementación; medidas de respuesta; países menos avanzados; agricultura; mercados; ACE; bosques; aspectos ligados a la ciencia; aspectos metodológicos bajo CMNUCC; género; otros temas de implementación del AP; LCIPP y marcos temporales comunes.

\section{Cobertura de la agenda climática de las COP por el equipo negociador de la $D A$}

Identificamos 8 equipos negociadores de la DA de 2012 a 2019, periodo que incluye momentos políticos clave a nivel internacional 
en la CMNUCC, como la adopción del Acuerdo de París en 2015 y del denominado Libro de Reglas de Katowice en 2018. Asimismo, a nivel de la política climática nacional, se identificaron los cambios de gobierno en 2015, tras 12 años de gestiones del mismo signo político, y en 2019 con un nuevo cambio luego de la presidencia de Mauricio Macri. De igual manera, se observaron otros momentos significativos durante este periodo como la presentación de la primera Contribución Determinada a nivel Nacional en 2016 y la creación del Gabinete Nacional de Cambio Climático mediante el Decreto No 891/2016, como la sanción de la Ley Nacional 27.520 de Presupuestos Mínimos de Adaptación y Mitigación al Cambio Climático Global en 2019.

De los 21 grupos construidos, 14 han tenido constante presencia en las agendas (azules o celestes en la figura 4) pero no necesariamente han sido cubiertos por el equipo negociador argentino. Ejemplos de cobertura relativamente constante del equipo negociador (color azul) son mitigación (8/8), agricultura (8/8), financiamiento $(8 / 8)$, adaptación $(7 / 8)$, pérdidas y daños $(7 / 8)$, transparencia $(6 / 8)$, metodología (6/8), medidas de respuesta (5/8) y tecnología (5/8). Cabe mencionar, respecto a los temas con amplia cobertura argentina, que en el periodo de estudio, el país ocupó el rol de coordinador líder del G77 más China hasta 2015 en el tópico "medidas de respuesta”, con la misma representante del área de negociaciones económicas de la cartera de relaciones exteriores. La cobertura del tópico por Argentina coincidió con los gobiernos de Cristina Fernández y el abandono se produjo con el cambio de gestión de Macri, quien consideró la cobertura del tema y mucho más su liderazgo en el Sur Global como un signo de la política climática del gobierno precedente. Las medidas de respuesta fueron tradicionalmente lideradas por Argentina y por Arabia Saudita, quienes buscaron el reconocimiento del impacto de las medidas de respuesta al cambio climático por los países desarrollados, especialmente en materia comercial, teniendo como foco de interés el sector agropecuario y el energético, respectivamente. Incluso el nuevo gobierno consumó la salida del país del grupo de negociación Like Minded Developing Countries (Bueno, 2020). 
Otro de los temas con alta cobertura que se ve reforzada por el liderazgo argentino del Sur Global en el G77 más China es el tema de adaptación. Argentina lideró el grupo antes del periodo de estudio de 2010 a 2011, incluyendo adaptación, pérdidas y daños en el AWG-LCA y también lo hizo de 2016 a 2018, exclusivamente sobre la agenda de adaptación ya escindida en el APA. Si bien el tema de adaptación comenzó siendo cubierto por el área ambiental, de la COP20 a la COP24 fue negociado por la cartera de relaciones exteriores en la misma persona de quien luego ofició como coordinadora líder del G77 más China y como copresidenta del Comité de Adaptación. En reconocimiento del liderazgo argentino en el tema, también el país cofacilitó negociaciones informales sobre pérdidas y daños en 2012 junto con Canadá y en 2018 con Australia (Bueno \& Siegele, 2020).

Se puede mencionar también el caso del grupo sobre metodología que tuvo una alta cobertura nacional dado que incluyó el tema bunkers o emisiones de los combustibles del transporte marítimo y aéreo. Este tópico ha sido tradicionalmente cubierto por el área de negociaciones económicas del Ministerio de Asuntos Exteriores como parte del paquete "comercio y cambio climático" (medidas de respuesta, bunkers y agricultura). Justamente respecto al tema agricultura, por la relevancia estratégica del tema para el país, este ha tenido constante cobertura, ya sea a través de la Cancillería exclusivamente, conjuntamente o mediante el área de agricultura. Cabe mencionar que Argentina no ha ocupado el rol de coordinador líder del G77 más China en este tema, en parte como una estrategia de menor exposición, pero ha oficiado como actor clave con Egipto, que muchas veces fue el titular. $\mathrm{Al}$ mismo tiempo, la alta prioridad del tópico se evidencia en que el cambio de gobierno no impactó la cobertura temática. Con respecto a la posición, que excede el objeto de este trabajo, cabe mencionar que si bien la misma no se modificó sustantivamente a nivel nacional en términos de la línea roja argentina en la mitigación agrícola, sí lo hizo la estrategia de abordaje. En este sentido, la posición permitió debatir aspectos ligados a la mitigación y exceder el tradicional debate exclusivo sobre adaptación del sector. 
Otro de los grupos con plena cobertura en el periodo fue el de financiamiento, aunque cabe mencionar que escasamente ha sido a partir de la representación del área económica (2/8), en la mayoría de los casos (6/8) fue negociado por la Cancillería. Esto da cuenta de la alta estabilidad en la relevancia del tópico, más allá de los gobiernos y de las áreas de gobierno que lo cubrieron. Este mismo interés no puede extrapolarse a otros medios de implementación, en la medida que la cobertura de tecnología estuvo ligada a la participación del representante experto que ocupó las presidencias del TEC y el CTCN. Desde el momento en el que dicho experto no participó más de las COP, Argentina no asignó un nuevo representante. En cuanto al tercer medio de implementación, construcción de capacidades, Argentina escasamente negoció el tema.

Cabe mencionar el grupo de transparencia, característico del mandato de Durban hacia París y uno de los temas política y técnicamente más sustantivos del periodo 2016 a 2019. Este tema comenzó con una cobertura del área de asuntos exteriores en el reconocimiento de su valor estratégico aunque con escasa traducción al plano nacional del reporte y seguimiento. Naturalmente, luego el tema fue cubierto por el área ambiental y especialmente por las representantes que efectivamente confeccionan los inventarios nacionales de gases de efecto invernadero (GEI), los informes bienales, las secciones de mitigación de las NDC y próximamente los informes de transparencia.

Es posible mencionar 4 temas más en los que el país asignó marginalmente negociadores/as como bosques $(2 / 6)$ y género $(1 / 7)$ o LDC y ciencia que no tuvieron cobertura. Es llamativo que el país no haya negociado activamente el tema bosques en vista de que la deforestación ubicó a Argentina entre los países con mayor nivel de pérdida de cobertura forestal y es una de las actividades más representativas en términos de emisiones de GEI del país. La ausencia de cobertura del tema en las COP23, COP24 y COP25 llama la atención a la luz de la presentación durante ese periodo del Primer resumen de información sobre salvaguardas de REDD+, el Nível de Referencia de Emisiones Forestales (NREF), el Anexo técnico REDD+ y la propuesta de pagos por resultados presentada y aprobada por el GCF en 
el 2020. En cuanto al tema género, es notable que recién haya tenido cobertura desde la última COP y es esperable que sea un nuevo tema de cobertura futura coincidente con la segunda NDC, que incorpora género dentro de los 15 ejes rectores que guían el diseño, implementación y monitoreo de todas las secciones (Ministerio de Ambiente y Desarrollo Sostenible, 2020).

Respecto a los temas ligados a los países menos desarrollados o LDC es relativamente razonable que ante recursos escasos en términos de negociadores/as, el país decida no atender temas que son de especial interés de los países que componen el grupo de LDC y de los países desarrollados que aportan apoyo de acuerdo a los compromisos diferenciados bajo la CMNUCC. En cuanto al grupo de ciencia, en todas las COP analizadas involucró los temas de observación sistemática e investigación en el SBSTA, incluso en la COP20 se incluyó la consideración sobre el Informe AR5 del IPCC y en la COP22 cómo las evaluaciones del IPCC deberían informar el proceso del balance mundial. A pesar de la gran relevancia de estos tópicos, Argentina no asignó prioridad a su cobertura.

Por otra parte, hay un conjunto de grupos cuyo origen se remonta a la implementación del Acuerdo de París desde 2016, como el balance mundial, el Comité para Facilitar la Implementación y Promover el Cumplimiento y los asuntos propios de la implementación del acuerdo; asimismo, desde la COP23 se adicionaron el tema common time frames a instancias de Brasil en el marco de la COP22 y la institucionalización de la LCIPP largamente negociada. En dichos temas y grupos, la cobertura argentina ha sido oscilante, donde temas propios del APA fueron en mayor medida negociados especialmente por la cartera de asuntos exteriores en el entendido que se trataba de los temas de mayor incidencia política en el corto plazo de su negociación específica. El tema y grupo common time frames, a partir de su establecimiento, fue recogido por las negociadoras del área ambiental a tono con su cobertura del grupo transparencia en el mismo periodo. Mientras tanto, el grupo y tema LCIPP no ha tenido cobertura nacional, lo que podría explicarse por la escasa transversalidad del tema "pueblos originarios" en la política climática. Es posible mencionar que no 
ha sido recogido por la Ley 27.520 ni por su Decreto Reglamentario 1030/2020. En contraposición, el tema figura en diversas oportunidades en la segunda NDC 2020.

Otro tema sin cobertura del equipo negociador argentino es el ACE que corresponde a un término adoptado en la CMNUCC para referirse a su artículo 6 y al artículo 12 del AP. Se busca empoderar a la sociedad mediante 6 áreas de trabajo de la acción climática: la educación, la capacitación, el incremento de la sensibilización, la participación, el acceso a la información y la cooperación internacional.

Finalmente, el tema mercados solo tuvo cierta cobertura durante la gestión de Mauricio Macri, en la cual había interés en involucrarse y participar del nuevo sistema de mercado del artículo 6 del AP, más allá de que no se lograron posiciones comunes en el marco del grupo ABU (Argentina, Brasil y Uruguay). De hecho, la primera NDC de 2016, involucró una mención explícita:

[...] cualquier transferencia de unidades de reducción de emisiones alcanzadas en territorio argentino deberá contar con la expresa autorización del Gobierno Nacional, y, salvo disposición expresa en contrario, todas las reducciones de emisiones en el territorio nacional serán contabilizadas para el logro de la meta de la NDC.

A pesar de que Argentina participó del Mecanismo de Desarrollo Limpio (MDL) en el marco del Protocolo de Kyoto, durante el periodo de estudio no se evidenció cobertura en la negociación de la materia. El informe de actualización bienal 3 del país (BUR3) da cuenta del registro de 46 proyectos bajo el MDL, 18 emitieron reducciones de emisiones certificadas (CER, por sus siglas en inglés). El informe también explicita la participación de empresas argentinas en mercados voluntarios, mencionando 6 proyectos en el Verified Carbon Standard (VCS). De 2006 a 2010 se registraron 4 proyectos anuales y en el periodo de estudio y específicamente en 2012 se alcanzó un pico de proyectos acreditados anuales de 16 en MDL, previo a la expiración del primer periodo de compromisos del Protocolo de Kyoto. Luego, el registro de proyectos bajó a cero a partir de 2016 (SAyDS, 2019). 
Figura 3. Cobertura de grupos climáticos por el equipo negociador argentino (COP18-COP25)

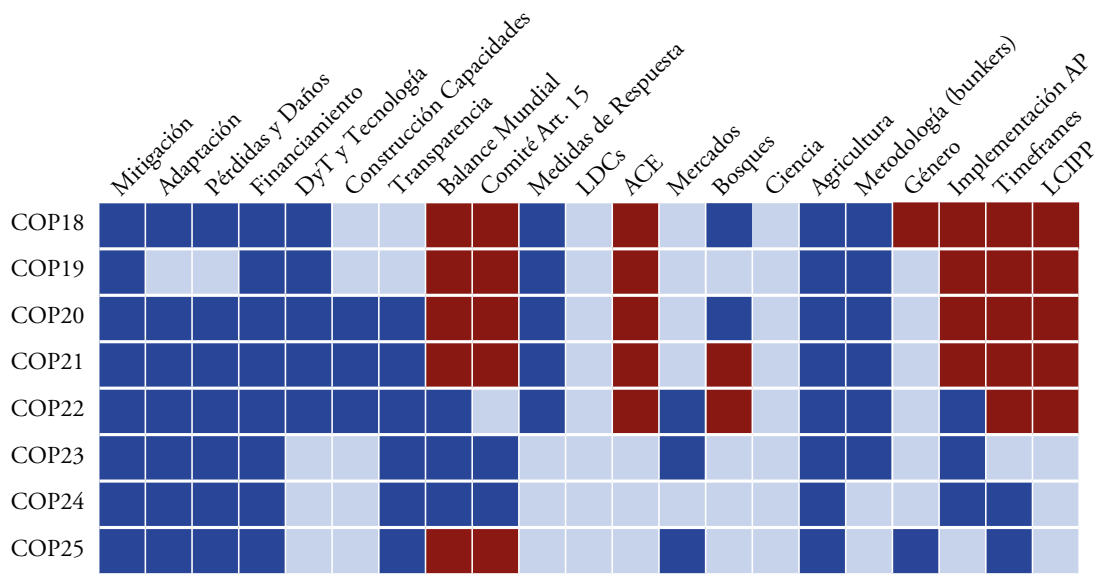

No en Agenda No Cubierto

Cubierto

Fuente: elaboración propia.

\section{Conclusiones}

A la luz de la literatura previa y la investigación realizada es posible afirmar en términos de aporte metodológico que el estudio sobre la composición y evolución de las delegaciones radica en la comparabilidad entre países con distintas condiciones económicas, políticas, sociales y de políticas climáticas a lo largo de distintos momentos del proceso negociador. Sin embargo, el número total de participantes en las delegaciones oficiales es sumamente variable a la luz de aspectos coyunturales con mayor o menor incidencia política: participación de presidentes o vicepresidentes; lugar en el que se realiza la COP; elecciones presidenciales y otros momentos claves de la política nacional; presentación de documentos internacionales (como NDC), y coyunturas internacionales y otros momentos claves de la CMNUCC, entre otros. Asimismo, existen aspectos estructurales relativos a la composición, incluída la disponibilidad de recursos económicos para financiar la participación, la trayectoria del país respecto al proceso negociador y la disponibilidad de recursos humanos capacitados, entre otros. 
Respecto a los equipos negociadores, esta investigación ofrece una innovación en relación con la bibliografía disponible y se estima clave avanzar en más estudios nacionales de esta índole que habiliten algún nivel de comparabilidad. Este tipo de enfoque ofrece la oportunidad de vincular a las delegaciones nacionales con los intereses de negociación no solo coyunturales sino estructurales y, por ende, con la política climática nacional. Asimismo, a pesar de no haber sido un objetivo de este trabajo, es el puntapié para construir relaciones entre la cobertura y el contenido de las posiciones nacionales.

En cuanto al caso de estudio, cabe mencionar que la DA se ha comportado en términos similares a otros Estados analizados en la literatura, en particular respecto a la preponderancia de las áreas de relaciones exteriores y ambiente, a las que puede adicionarse el caso de energía y, particularmente, agricultura. Entre ambas carteras se encuentra el 90\% de las emisiones argentinas de GEI, aunque el accionar de ambas ha sido disímil en la medida que la mitigación sectorial en agricultura es la principal línea roja de la posición nacional. En consecuencia, el tema agricultura ha tenido cobertura constante ya sea del área de negociaciones económicas de la Cancillería o de la cartera propia de agricultura.

A diferencia de otros casos que figuran en la literatura, la DA ha experimentado un incremento en el número de participantes tanto de la COP1 a la COP25 como en el periodo de estudio. Sin embargo, esos incrementos se han dado por debajo del promedio de las otras delegaciones y ha tenido una conducta errática con picos ligados a las características coyunturales nacionales.

El equipo negociador argentino es pequeño con relación al tamaño total de la DA. No obstante, este contó con cierta estabilidad en el periodo estudiado. Esto significa que muchos/as de los/as negociadores/as participaron en varias COP. El área de relaciones exteriores ha liderado al equipo negociador pero se ha visto parcialmente retraído a favor de la cartera ambiental que ha ocupado roles que naturalmente deben negociar los técnicos que elaboran los documentos nacionales, incluyendo los sistemas de reporte. Esto ha estado acompasado con los cambios en el proceso de la CMNUCC donde la complejización 
técnico-política ha ido en aumento, teniendo a las negociaciones de transparencia como caso.

En cuanto a la cobertura temática y por grupos, es posible identificar tendencias dentro de las que se presentan temáticas de alta, media y baja cobertura. Dentro de las primeras se puede mencionar: agricultura, mitigación, adaptación, pérdidas y daños, financiamiento, transparencia, metodologías por bunkers, balance mundial, Comité del artículo 15 del AP, implementación del AP y common time frames. Respecto a los tópicos de cobertura media, se mencionan tecnología y medidas de respuesta, en los que se evidencian oscilaciones en la posición y en quienes negocian. Finalmente, se destacan aquellas de baja o nula cobertura: bosques, género, mercados, capacidades, LDC, ACE, ciencia y LCIPP.

En adición al aporte del conocimiento de la participación argentina en las negociaciones climáticas, el presente trabajo contribuye a la comprensión de la conformación y composición de las delegaciones, los equipos negociadores y la cobertura de la agenda, hecho que se esgrime particularmente relevante para los Estados en desarrollo, quienes deben hacer frente a la creciente complejización de la dinámica negociadora en el tópico, la proliferación de temáticas dentro de la agenda climática y al aumento del peso político del cambio climático en la arena internacional. En este sentido, la propuesta de analizar a los equipos negociadores y observar en qué espacios de la negociación participan puede generar información valiosa sobre las políticas exteriores climáticas de Estados que no tienen la capacidad de participar activamente en todos los temas en discusión dentro de cada COP. En este sentido, se plantea a futuro la necesidad de seguir profundizando sobre el proceso explicativo, así como vincular los temas y grupos cubiertos con la política climática doméstica, de modo que se pueda alcanzar una comprensión más acabada del funcionamiento de los equipos negociadores y del alcance y profundidad de la cobertura. 


\section{Referencias}

Abbott, K. (2017). Orchestration: Strategic ordering in polycentric climate governance. http://dx.doi.org/10.2139/ssrn.2983512

Andonova, L. B., Betsill, M. M., \& Bulkeley, H. (2009). Transnational climate governance. Global Environmental Politics, 9(2), 52-73. https://doi. org/10.1162/glep.2009.9.2.52

Argentina, Ministerio de Ambiente y Desarrollo Sostenible. (2020). Segunda contribución determinada a nivel nacional. https://www4.unfccc.int/sites / ndcstaging/PublishedDocuments / Argentina\%20Second/Argentina_Segunda $\% 20$ Contribuci $\%$ C $3 \%$ B3n $\% 20$ Nacional.pdf

Argentina, Gobieno de la República. (2016). Primera contribución determinada a nivel nacional. https://www4.unfccc.int/sites/ndcstaging/PublishedDocuments/Argentina\%20First/17112016\%20NDC\%20Revisada \%202016.pdf

Bailer, S. (2017). Bargaining resources and strategies in climate change negotiations. Workshop Negotiating Climate Change, Zurich: Center for Comparative and International Studies (CIS). https: / / snis.ch/wp-content/ uploads/2019/12/2009_Michaelowa_Working-Paper-4.pdf

Betzold, C. (2010). 'Borrowing' power to influence international negotiations: AOSIS in the climate change regime, 1990 - 1997. Politics, 30(3), 131-148. https://doi.org/10.1111/j.1467-9256.2010.01377.x

Bhandary, R. R. (2017). Coalition strategies in the climate negotiations: An analysis of mountain-related coalitions. International Environmental Agreements: Politics, Law and Economics, 17(2), 173-190. https:/ / doi. org/10.1007/s10784-015-9313-6

Böhmelt, T. (2013). Civil society lobbying and countries 'climate change policies: a matching approach. Climate Policy, 13(6), 698-717.

Bueno, M. P. (2020). Algunas aproximaciones a la política climática de Cambiemos (2015-2019). En E. Iglesias \& J. B Lucca (Ed.), Algunas aproximaciones a la política climática de Cambiemos (2015-2019) (pp. 189203). UNR Editora.

Bueno, M. P. (2012). Los vaivenes de la diplomacia climática argentina (1989-2011). En C. Morasso, \& G. Pereya Doval (Comp.), Argentinay Brasil: proyecciones internacionales, cooperación Sur-Sur e integración (pp. 134154). UNR Editora. 
Bueno, M.P., González, J., \& Yamin, P. (2021). Delegaciones argentinas en las conferencias de las partes de la Convención Marco de Naciones Unidas sobre Cambio Climático: Evolución numérica, composición y género. Argentina $1.5^{\circ} \mathrm{C}$. https://unr.edu.ar/noticia/14457/arg15-informe-de-politica-nro-5

Bueno, M. P., \& Yamin Vázquez, P. (2017). ¿Poder material o poder social? Reflexiones sobre las negociaciones multilaterales y el poder climático de la Argentina (2003-2015). Relaciones Internacionales, 26(53), 65-86.

Bueno Rubial, M. P., \& Siegele, L. (Eds.). (2020). Negotiating climate change adaptation. The common position of the Group of 77 and China. Springer International Publishing. https://doi.org/10.1007/978-3-030-41021-6 Bulkeley, H., Andonova, L., Backstrand, K., Betsill, M., Compagnon, D., Duffy, R., Kolk, A., Hoffmann, M., Levy, D., Newell, P., Milledge, T., Paterson, M., Pattberg, P., \& VanDeveer, S. (2012). Governing climate change transnationally: assessing the evidence from a database of sixty initiatives. Environment and Planning C: Government and Policy, 30(4), 591-612. https://doi.org/10.1068/c11126

de Águeda Corneloup, I., \& Mol, A. P. J. (2014). Small island developing states and international climate change negotiations: the power of moral "leadership". International Environmental Agreements: Politics, Law and Economics, 14(3), 281-297. https:/ / doi.org/10.1007/s10784-013-9227-0

Fisher, D. R., \& Green, J. F. (2004). Understanding disenfranchisement: Civil society and developing countries' influence and participation in global governance for sustainable development. Global Environmental Politics, 4(3), 65-84. https://doi.org/10.1162/1526380041748047

Intergovernmental Panel on Climate Change [IPCC]. (2018). Summary for Policymakers. In: Global warming of $1.5^{\circ} \mathrm{C}$. An IPCC Special Report on the impacts of global warming of $1.5^{\circ} \mathrm{C}$ above pre-industrial levels and related global greenhouse gas emission pathways, in the context of strengthening the global response to the threat of climate change, sustainable development, and efforts to eradicate poverty. World Meteorological Organization. https://www. ipcc.ch/sr15/chapter/spm/

González, J. H. (2018). Vaivenes en la política climática y forestal Argentina: La vinculación entre el país y el mecanismo REDD+. Revista Chilena de Relaciones Internacionales, II(2), 105-121. 
Grubb, M., \& Yamin, F. (2001). Climatic collapse at The Hague: What happened, why, and where do we go from here? International Affairs, 77(2), 261-276.

Joshi, S. (2015). North-South relations: colonialism, empire and international order. En P. G. Harris (Ed.), Routledge handbook of global environmental politics (pp. 272-283). Routledge. https:/ /doi.org/10.4324/9780203799055

Kaya, A., \& Schofield, L. S. (2020). Which countries send more delegates to climate change conferences? Analysis of UNFCCC COPs, 1995 - 2015. Foreign Policy Analysis, 16(3), 478-491. https://doi.org/10.1093/fpa/ orz031

Kruse, J. (2014). Women's representation in the UN climate change negotiations: a quantitative analysis of state delegations, 1995 - 2011. International Environmental Agreements: Politics, Law and Economics, 14(4), 349-370. https://doi.org/10.1007/s10784-014-9245-6

Lövbrand, E., Hjerpe, M., \& Linnér, B. O. (2017). Making climate governance global: How UN climate summitry comes to matter in a complex climate regime. Environmental Politics, 26(4), 580-599. https://doi.org /10.1080/09644016.2017.1319019

Martinez, G. S., Hansen, J. I., Olsen, K. H., Ackom, E. K., Haselip, J. A., Bois von Kursk, O., \& Bekker-Nielsen Dunbar, M. (2019). Delegation size and equity in climate negotiations: An exploration of key issues. Carbon Management, 10(4), 431-435. https://doi.org/10.1080/175830 04.2019 .1630243

Ourbak, T., \& Magnan, A. K. (2018). The Paris Agreement and climate change negotiations: Small islands, big players. Regional Environmental Change, 18(8), 2201-2207. https:/ / doi.org/10.1007/s10113-017-1247-9

Programa de Naciones Unidas para el Medio Ambiente [PNUMA]. (2020). Emissions Gap Report 2020. https://www.unep.org/es/emissions-gap-report-2020

Programa de Naciones Unidas para el Desarrollo [PNUD]. (2021). Adaptation Gap Report 2020. https://www.unep.org/es/resources/informe-sobrela-brecha-de-adaptacion-2020

Roger, C., \& Belliethathan, S. (2016). Africa in the global climate change negotiations. International Environmental Agreements: Politics, Law and Economics, 16(1), 91-108. https:/ / doi.org/10.1007/s10784-014-9244-7 SAyDS (2019). Tercer informe bienal de actualización de Argentina a la Convención Marco de las Naciones Unidas para el Cambio Climático (CMNUCC). https:/ / 
unfccc.int/sites/default/files/resource/3er\%20Informe $\% 20$ Bienal $\% 20$ de $\% 201 \mathrm{a} \% 20$ Republica $\% 20$ Argentina.pdf

Schroeder, H., Boykoff, M. T., \& Spiers, L. (2012). Equity and state representations in climate negotiations. Nature Climate Change, 2(12), 834836. https://doi.org/10.1038/nclimate1742

Skovgaard, J., Ferrari, S. S., \& Knaggård, A. (2019). Mapping and clustering the adoption of carbon pricing policies: What polities price carbon and why? Climate Policy, 19(9), 1-13. Doi: https://doi.org/10.1080/1 4693062.2019.1641460

Stevenson, H., \& Dryzek, J. S. (2014). Democratizing global climate governance. Cambridge University Press.

Weiler, F. (2012). Determinants of bargaining success in the climate change negotiations. Climate Policy, 12(5), 552-574. https://doi.org/10.1080 /14693062.2012.691225 